\title{
II. Buchbesprechungen
}

W. StoeekeL Lehrbuch der Gynäkologie. II. völlig neubearbeitete Auflage mit 479 schwarzen und farbigen Abbildungen im Text und auf 64 farbigen Tafeln. Leipzig 1928, S. Hirzel. 46.- Mk. Es ist ein hocherfreuliches und nicht genug zu würdigendes Verdienst deutscher Autoren und Verleger, daß sie trotz der drückenden Not der Zeit keine Mühen und Kosten seheuen, unseren wissenschaftlichen Bücher-markt nicht nur auf der bisherigen Höhe zu erhalten, sondern, vorwärts strebend, immer besser auszugestalten, zu vervollkommnen und der Welt zu zeigen, daß deutscher Geist und deutsche Kunst unter Krieg und Revolution nicht gelitten haben. Solche Gedanken bewegen uns, wenn wir Stoeckels jüngstes Werk über-schauen, die von iha $\alpha$ völlig neubearbeitete zweite Auflage seines ,,Lehr-buches der Gynäkologie”, ein stattliches Buch, das in seiner gründlichen Durcharbeitung des umfangreichen Stoffes einschließlich aller Grenzgebiete eine staunênswerte Leistung darstellt. Wie würde sich Heinrich Fritsch, dessen Gedächtnis das Buch gewidmet ist, freuen, wenn er hätte erleben können, wie ausgezeichnet sein wissenschaftliches Erbe von seinem ihm auch persönlich so nahegestandenen Schüler bewahrt und fortgebildet

238

Buclibesprechungen.

wird: ,,Zum Andenken an den Mann, der zu den hervorragendsten Führern in der Gynäkologie gehört hat", mit welchen Worten Stoeckel selb $1 / 8$ t in der Vorrede der ersten Auflage seiner pietätvoll gedenkt.

Vor mir liegt die ,III. vermehrte und verbesserte Auflage” der ,Krank-heiten der Frauen” von Fritsch aus dem Jahre 1886, ein für die damalige Zeit ganz ausgezeichnetes Buch, aber anspruchslos in Umfang und Abbil-dungen, konnte er doch auf 484 Seiten den ganzen Stoff bewältigen, und die 150 Abbildungen in Holzschnitten zeigen, so gut sie sind, eben doch vielfach, besonders in den mikroskopisch-anatomischen Darstellungen recht bescheidene Ansprüche. Und was hat Stoeckel nun daraus gemacht! Der Umfang des natürlich ganz neuen Buches ist in Anbetracht des größeren Formates wohl auf das Doppelte gestiegen; zahlreiche neue Kapitel be-zeugen, wie unsere Gynäkologie immer weitere Kreise zieht und die geradezu glänzende Ausstattung des Buches, besonders in den vielen Abbildungen im Text und in farbigen Tafeln, stellt ein Meisterwerk der Illustrations-kunst dar. Freilich gehört für die richtige Herstellung und Auswahl der Bilder dazu auch der Geist des Autors. Zuna großen Teile sehen wir ganz vorzügliche Originale, zum anderen, wesentlich geringeren, eine sehr ge-lungene Auswahl aus der Literatur, ebenso anschaulich als schön!

Die erste Auflage aus dem Jahre 1924, die noch als „,völlig neube-arbeitete 13. Auflage” des Lehrbuches von Fritsch bezeichnet war, hat Stoeckel mit dem inzwischen leider so früh verstorbenen Reifferscheid ver-faßt. Sein Tod stellte Stoeckel vor die Frage, die neue Auflage entweder nun mit einern anderen Mitarbeiter z $\backslash$ verfassen oder allein die gewaltige Arbeitslast zu übernehmen. Er wählte das letztere und traf ganz gewiß, wie der Erfolg zeigt, damit das Richtige. Ich fühle ihm dies lebhaft nach, weil ich ja durch den Tod Krònigs vor Jahren in das gleiche Dilemma gebracht worden war, und es mögen wohl auch die gleichen Überlegungen 
gewesen sein, die Stoeckel dabei bewegten. Auch beim bestenEinvernehmen zwischen zwei Autoren entstehen bei der Abfassung soldier Bücher doch manchmal mehr oder weniger Schwierigkeiten: denn gliicklicherweise können auch Gleichgestimmte nicht immer Gleichgesinnte sein. Die Wissen-schaft ware ja langweilig, wenn man in alien Dingen nur einer Meinung ware. So muß man eben in den zahlreichen Besprechungen, die zwei Ver-fasser solcher Werke inszenieren, manche Kompromisse schließen. Erst wenn man dann allein den ganzen Stoff an sich voriiberziehen läßt und be-arbeitet, kann man frei schalten und walten, wobei es noch eine offene Frage ist, ob nicht die Kompromißarbeit für die Leser interessanter und lehrreicher ist. Lehrbücher dürfen ja nie allzu subjektiv gehalten werden, sollen keine Monographien sein, ein beklagenswerter Fehler mancher ISГ euerscheinungen, wie das jetzt beliebt wird. Ich habe die Erfahrung gemacht, daß gerade harmonisches Zusammenarbeiten vor einer Überschätzung der eigenen Meinung recht schützt. So hat alles auch hier seine zwei Seiten;

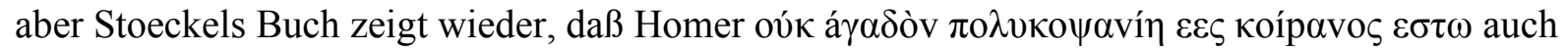
beim Bücherschreiben seine Berechtigung hat. So ist dieses neueste Lehrbuch der Gynäkologie aus einem $\mathrm{Gu} ß$ geschrieben, in ganz ausgezeichnetem, vorbildlichem Stil und klarer Darstellung. Man gewinnt beim Durchsehen der einzelnen Kapitel den Eindruck, daß weder Verfasser noch Verleger jemals sich von anderen Rücksichten leiten

I $>$ uchbesprechungeñ.

239

ließeu, a Is die vollkommenste und beste Darstellung mid Ausstattung zu geben.

Die ersten Kapitel führen durch topographische Anatomie, Physiologie mid

Entwicklungsgeschichte der weiblichen Genitalien in das Gebiet ein, wobei besonders atich die neueren Forschungen über das Beckenbindegewebe von /:. Martin in großen Bildern eindrucksvoll berücksichtigt sind. An-schließend daran kommen die Mißbildungen der weiblichen Genitalien.

Die Überleitung zvm klinischen ïeil bringt die gynäkologisehe Dia-gnostik, in die sehr geschickt bei der äußereii Untersuchung auch die Kon-stitutionstypen als ganz modemer Abschnitt eiiigeschaltet sind. Den Kapiteln über die Erkrankungen der Vulva und Vagina folgt ein ausführ1 idles über die Erkrankungen der weiblichen Harnorgane, ein Gebiet, auf dem ja Stoeckcl führend wurde.

Welch wichtige Umwälzungen einer der bedeutungsvollsten Vorgänge ini weiblichen Leben durch die neueren Forschungen erfahren hat, ist im VIII. Kapitel über Menstruation, und Menstruationsanomalien klipp und klar (largest el It. Síoeckel versteht es ausgezeichnet, alles überflüssige Bei-werk wegzulassen, und wer sich mit möglichst wenig Mühe über diese neueren Errungeiischaí'ten miseres Faches orientieren will, kann keine klarere Darstellung finden als in diesem Buche. Bei der Bezeichimng der verschiedenen Arten und Abarten der Menstruation möchte ich < ier von Seitz (Zbl. f. G. 1922, S. 50) den Vorzug geben. Ich finde, daß die von Seitz eingeführten Bezeichnungen ,,Opsoxienorrhöe, Proiomenorrhöe” und dann auch die Unterscheidiuig zwischen ,,Algomenorrhöe” und ,Dysmenorrhöe” treffen-der sind als die althergebrachten, die auf diese verschiedenartigen Zeitfolgen und CJnterscheidung zwischen allgemeinen und- örtlichen Beschwerden keine Rücksicht nehmen.

Audi im folgenden Kapitel, das von den Entzündungen des Uterus liandelt, mußte diesen neuen. Forschungen über die Veränderungen der Uterusschleimhau†', die fälschlicherweise früher als Entzündungen gedeutet wurden, während sie jetzt als normale zyklische Veränderungen ânerkamit sind. Redlining getragen werden. 
Bei den Lageveränderungen des Uterus halt Stoeckcl mit Recht noch daran fest, daß es nicht ganz gleichgültig ist, wie der Uterus liegt, wie manche über das Ziel Hinaussc'hießende uus vormachen wollen. Er behandelt des-halb das Kapitel uach dem. klassischen Muster von Sclmltze und Fritsch und gibt auch den 1/1/8geverändernden Operationen ihr Recht.

Xach dei- großen Bedeutung, die die Myome nach Zahl mid iSchwere ihres Auftretens verdienen, widmet diesen so vielgestaltigen Erscheinmigen und Formen Stoeckel eingehende Darstellung in Text und Bild. Nicht leicht ist es ja heute, die Therapie der Myome zu schildern. Die symptomatische Behandlung verwirft er und alle die früheren, harmlos erscheinenden und doch so gefährlichen Eingriffe, wie die $\Lambda$ po.sío/ísche Galvanisation; ja selbst die medikamentöse Behandlung wird mit Recht abgelehnt, ebenso die Tainponadebehaiidlung, alles Verfahren, die eben doch auf die Dauer unbefriedigend sind. Bestrahlen oder 0|)erieren ist im einzelnen Falle nicht leicht zu entscheiden. Stoeckel rat, es den Myomtherapeuten zu überlassen, miter denen ja manche mehr Neigung zu dem einen, manche zu dem anderen Verfahren haben. Die Beurteilung der Bestrahlung bei den Myomen kann Monatsschrift f. Geburtshülfe u. Gynäkologie. Bd. LXXX. Heft 2,;i. 240

Bueh be spree hunger).

ich nielli- ganz so auffassen wie Stoeckel. Ich finde, daß eben doch die theoretisehen Bedenken, die ja schon Fritsch bei der Kastration. hatte, wo er das Wort prägte: ..Man entfernt die gesunden Organe und läßt das Kranke zurück", zu doktrinär sind. In. diesen rein, praktischen Fragen entscheidet der Erfolg, und meine große Erfahrung geht dahin. daí3 selbstverständlieh nicht alle, aber immerhin die größere Mehrzahl der deiri klimakterischen Alter nahestehendc n Fran en und um solche handelt es sieli ja meistens bei den Myomkranken - (lurch richtige Bestrahlung in gänzlich gefahrlosei Weise von ihren Beschwerden befreit werden. Selbst werni die Myome nicht verschwinden. was wir ja immer wieder als Überraschtmg sehen, bleiben die Trägerinnen doch die fernere Zeit ihres Lebens vollkommen befreit von alien Krankheitserseheinungen, wenn auch die Krankheit noch im Körper isl. Ungünstige Spätfolgen sehen wir bei der Bestrahlung weder im allgemein, en noch im örtlichen Befinden. Aber es ist richtig, daß man sehr vorsichtig auswählen muß, namentlich bei jüngeren Fraurn. und daß für diese die vef schiedenartigen Operationsmethoden, die Stoeckel eingehend bespricht $1 / 8$ in Betracht kommen.

Auch in. der Behandlung der Epithelialtuinoren des Uterus, die das näehste Kapitel bringt, stehe ich auf einem etwas anderen Standpunkt als Stoeckel, insofern als ich mich von der Operation abgewandt habe und nur mehr bestrahle und von Jahr zu Ja.hr ein überzeugterer Anhänger dafür werde, daß die Bestrahlung das Feld der Zukunft behaupten wird. Es ist gut, daß wir nicht alle an einem Strang ziehen. Stoeckel bevorzugt noch die Operation, und zwar die vaginale. und gönnt ihr deshalb einen. breiten Raum; aber ich habe mich um so mehr gefreut, auch hier seine Objektivität anerkennen zu düríen. Er gibt eine gute Übersicht über den gegenwärtigen Stand der Strahlenbehandlungsresultate, und wie sehr er der Strahlen-behandlung zu ihrem Recht verhelfen will, geht daraus hervor, daß das folgende Kapitel von seinem Assistenten, Privatdozenten Dr. ü. Mikulicz-Radecki, bearbeitet ist: ,,Die Strahlenbehandlung in der Gynäkologie”, in dem in kurzer, aber vollkommen. genügender und erschöpfender Dar-stellung das Wesentliche der Strahlenbehandlung enthalten ist.

Die Krankheiten der Ovarien stellen eines der abgeklärtesten Kapitel der Gynäkologie dar, deren Behandlung koine Schwierigkeiten bietet. anders das folgende Kapitel, Erkrankungen. der 
Adnexe, wo die Gonorrhöe und die Tnberkulose der Genitalien eingehend berücksichtigt werden.

Sehr anziehend sind dann die nächsten Kapitel über Konzeption, Sterilität, Sterilisierung und dann die Grenzgebiete, die Erkrankungen der Briiste, der Banchdecken und die Darmleiden. Die drei letzten Kapitel, die in der vorigen Auflage Reiiierseheid bearbeitet Iiatte, sind. nun von Stoeckel ganz neu und vorzüglich dargestellt, so besondtrs das der Hygiene, in der er in. unterhaltsamer Weise die modischen Answüchse vom Standpunkt des Fraueuar/,tts bekämpf $†$. Den von Fritsch eingeführten Aiü^iang der Pharmacopoea gynaecologica hat auch Stoeckel beibehalten und mit alien Neuerimgen vervollständigt. Möchten nicht nur die deutschen Gynäkologen in diesem vortrefflichen Werke Belehrung, wissenschaftlichen und praktischen Gewinn suchen und finden und möchte ein rascher Umsatz auch dieser Auflage vielen folgenclen zu neuem Leben verhelfen! A. Döderlein-München.

Buchbesprechungeti.

211

Пeiilschcr (íynäkolo³/8en-Kalender 1928. Zentralblatt t'ür (Jynäkvlvgie, Erg1/8nzungsband. F. Michelson und B. Stoeckcl. Leipzig 1928. J. A. Barth. Bio- und Bibliolvgisehes Vev/.eichnis der deu†schen Frauenärzte.

In Analogic des KaJenders der deutsehen Chirurgen bringt die Verlags-bxichhandlung diesen hoehinteressanten Kalender, der miter der Mit-wirkung von Stoeckcl unû Michelsohn mit. großer Sorgfali herge stellt worden ist. Es werden die Personalien gebracht, wie Gebxirtstag, Studiengang und literarische Leistungen, dann in einer Liste die Direktoren der I $\tau n i-v$ 'ersitätsFraneiiklinik $\theta \prod$ und die Direktoren der gynäkologischen Ab-teilxmgen der öffentliehen Krankenhauser, eiidlioh ein Ortsverzeichnis. Wir begrül,îen dieses Werk als eine ausgezeielmete Гnterstüt/.ung. die in jedes Frauenarztes Hand sein sollto.

I $>$ ei der Herstollung dcrartiger Kalender kann tuan zwoi Woge vert'olgen: entweder der Ver†' asker versendet an alle in Frage Kommenden entsprechende Schemata, die er dann zusammenstellt: so hat die Firuia Degener es gemach.t mit ihrem ,.Wer ist's ?" Dann ist jeder sohuld daran, wenn er gar nicht oder uurichtig antwortet. Oder alle Zxxgehörigen des betreífenden Beru†'s werden in Zeitschriften znr Beteiligung aufgeiufen. Dann koirnnt es darauf an, diesen L·ockrnf uicht zu übersehen und ihm zn t'olgen. Herr Michclsohn hat diesen letzten Weg betreten. Wer ihm nicht gefolgt ist, darf sich iiber die Folgen nicht ärgern. Der Referent gehört zu der letzten Oruppe. Seinen Leidensgenossen kann er zxxm Trost sagen, daß eine neue Auflage in nicht allzu ferner Zeit in Aussicht steht, so daß wir doch auch noch drankommen: wenn wir es nämlich erleben! Jedenfalls begrüße ich diese Ergänzung unserer Literatur atif das lebhafteste, ebenso wie den in Aussicht stehenden Registerband über den Inhalt der ersten 50 Jahrgänge des Zeniralblatts. $\Lambda$. Martin. Werner Kftrté. Die Clüлirgic des Peritoneum. Neue deutsehe Chirurgie. 39. Bú. Verlag Enke. Stuttgart 1927.

Der anerkannte Pionier in $(\backslash<\tau$ Entwieklung der peritonealen Chirurgie gibt in klassischer Dai'stellung die Sunime seiner Frfahrungen auf diesem seinem eigensten (1/8biete. Mul.i das sclion allein. für uns Frauenärzte von großer Bed exit ung erscheinen, so erliöht sich diese (lurch den Гnistand, daß Kòrte. ein außerordentlich großes Material auch aus unserem Fach $\mathrm{v}^{3} / 8 \Lambda$ hrend 
seiner rnehr als 35jiíhrigon Tätigkeit am IJrbankrankenhaus und einer weitreichenden Privatpraxis zu beobachten und zu bearbeiten (íelegenheit, gehabt hat.

Xach einetn liistorisclien íborblick über die Entwieklung der Chirurgie des Peritoneum gibt Yerf. miter Beifugung entsprechender Literatur eine klare Darstellung von der Anatoxnie und Physiologie, dann von der pathologischen Anatomic, ihren Ausgaiigspunkten und Vorlauf. Eingehend werden die Symptome erörtert.

An das Kapitel über die chirurgischo Bohandlung reiht sich an eine Erörterung der Besonderheiten einzelner For men der akxiten und der chro-nisehen Bauchfellentzündxxngen. Es schließen sich an die Kapitel iiber Staumigsaszi†es. (íeschwülste und Verletzungen.

Für mxs ist es von besondorem Wert zu verfolgen, wie sich die Behand-lung auch derjenigen Formen $\left(\mathrm{i}^{\wedge} \mathrm{v}\right.$ Banchfellentzündxxng entwickelt bat.

$1(5 *$

242 Buchbesprechungeu - Luigi Matigiagalli $\uparrow$.

die wir als von den weiblichen Genitalien ausgehend als unsere beson-dere Domäne zn beobachten haben. Während einer ersten Periode, dem Material von 20 Jahren, ist es Körte gelungen, nur 29,1 \% am Leben zu ßrhalten, in einer zweiten Periode 1911-1924 komvte er 65,9 $\%$ rotten, naehdem er die Frühoperation durehgeführt. Dabei bilden die puerperalen Fälle die auch in dieser Zeit am intensivsten gefährdete Gruppe.

Ref. beschränkt sicl·i absichtlich auf diesen knrzen Nacbweis, vim den Leser zu eigeiiem eingehendem Studium anzuregen. Dem Herrn Verf. sprioht Ref. den Wunsch aus, daß bei einer eventuellen neuen Auflage die Peritonisation nach seinen eigenen Erfahrungen eine eingehendere Würdigung finden möchte. A. Martin.

Luigi Mangiagalli $\uparrow$.

In Mailand ist anfangs dieses Monats (Juli 1928) einer cler einfluß-reichsten Führer der modernen Frauenheilkunde in Italien gestorben! Mangiagalli war ein typiseher Norditaliener: hochintelligent, vollei· Ideen, deren Verwirklichung er mit unermüdlieher Kraft durchsetzte! Ein Schüler von Fibone-Tnr'm tmd Calderini-Bologna besuchte er vor seiner S $\theta$ ßhaftmachung Paris mid Berlin. Er machte mir in seineu mehrwöchigen Arbeiten an meiner Seite den Eindruck eines Mamies von ungewöhnlichem Wissen und unentwegtem Können.

Früh $\Lambda$ vnrde il $\pi$ n die Entwicklung der gynäkologischen Klinik in Mailand anvertraiit: er hat sie zu einem vveithin wirkenden Zentrum ent-wick $\theta 1 t$ ! Er hat die Klinik, der er freigebig aus seinen eigenen Mitteln aufhalf, liis vor wenigen Jahren geführt und ausgestaltet.

Seine unerschöpfliohe Tatkraft ließ ilm, den glühenden Patrioten, auch in Stadt mid Staat $1>$ edeutungsvolle Aufgaben übernehmen. Er war einer der ersten faszistischen Senatoren in Rom und Biirgermeister von Mailand, das er in seiner stolzaufstrebenden Entwicklung im Wettstreit mit Rom führte!

Mangiagalli war ein lebhafter Mitarbeiter bei dem Streben nach internationalem

Zusammenschluß unseres Faches. Mit Jacobs und dem Uiiterzeichiïten wurde er zum

Treuhänder des Kapitals der internationalen gynäkologischen Gesellsehaft gewählt. Nach dem vorzeitigen Tod von Jacobs habe ich in Übereinstimmung mit Mangiagalli diesen Schatz, der in einer Brüsseler Bank ruht, der Belgischen gynälcologischen Gesellsehaft anvertraut.

Mangiagallis wissenschaftlichen Leistungen liegegnen wir aid' alien (1/8bietes unseres Faches: er war seinerzeit auch Mitarbeiter dieser Monats-schrift. Bewunderungswürdig polyglott, ein sehr gewandter Redner, war er auf alien Gebieten von Wissenschaft und Kunst orientiert. 
Ala,ngiagallis Name wird in Mailand durch seine Stiftungen festgehalten bleiben. Mit seinen zahlreichen Scliülern und Freunden bewahre ich ihm ein treues Andenken ! A. Martin. 